Forum 2021 · 36:167-170

https://doi.org/10.1007/s12312-021-00903-6

Online publiziert: 26. Februar 2021

(c) Springer Medizin Verlag $\mathrm{GmbH}$, ein Teil von Springer Nature 2021
Nachdem die erste Welle der CoronaPandemie im Sommer 2020 in Deutschland abgeflacht war, initiierten wir eine Erhebung zu den Erfahrungen von Psychoonkologinnen (männliche Kollegen im Folgenden immer eingeschlossen) zum Umgang mit den Corona-Beschränkungen und um daraus mögliche Konsequenzen abzuleiten. Folgende Fragen standen im Vordergrund:

Wie ist es Menschen mit einer Krebserkrankung in den Zeiten der strengen Kontaktbeschränkungen Anfang des Jahres aus Sicht der Psychoonkologinnen ergangen? Was haben professionelle Berufsgruppen erlebt, die an Krebs Erkrankte begleiten? Was können wir daraus lernen?

\section{Methoden}

Im Rahmen der Arbeitsgruppe Psychoonkologie der Arbeitsgemeinschaft

\begin{tabular}{|ll}
\hline Autor & \\
\hline & $\begin{array}{l}\text { Dr. Dipl.-Psych. } \\
\text { Andreas Ihrig } \\
\text { Universitätsklinikum } \\
\text { Heidelberg, Heidelberg }\end{array}$ \\
\hline
\end{tabular}

Sabine Götz ${ }^{1}$ für AG Psychoonkologie der ATO und Qualitätsverbund Krebsberatungsstellen in BW · Andreas Ihrig für AG Psychoonkologie der ATO und Qualitätsverbund Krebsberatungsstellen in BW

'Psychosoziale Krebsberatungsstelle, Sigmaringen, Deutschland

${ }^{2}$ Psychosoziale Krebsberatungsstelle Nordbaden, Sektion Psychoonkologie, Klinik für Allgemeine Innere Medizin und Psychosomatik, Universitätsklinikum Heidelberg, Heidelberg, Deutschland

\title{
Psychoonkologie während der Zeit der ersten restriktiven Corona-Beschränkungen
}

\section{Ergebnisse einer Befragung von 54 Psychoonkologinnen aus Onkologischen Zentren und Krebsberatungsstellen in Baden-Württemberg}

der Tumorzentren und onkologischen Schwerpunkte und Arbeitskreise (ATO) sowie dem Qualitätsverbund der Krebsberatungsstellen in Baden-Württemberg wurden psychoonkologisch tätige Mitarbeiterinnen per E-Mail angefragt, an der Studie teilzunehmen. Die Fragebögen von 54 Psychoonkologinnen aus der stationären und ambulanten Patientenversorgung in Baden-Württemberg konnten in die Auswertung einbezogen werden.

Zur Erfassung der Erfahrungen stellten wir einen Selbst- und Fremdbeurteilungsfragebogen zusammen. Er besteht aus offenen Fragen zu den persönlichen Erfahrungen (Wie ist es Ihnen ganz persönlich bei der Arbeit während der Krise ergangen? Inwiefern haben sich Ihre Beratungen verändert? Welche Rolle spielt in den Gesprächen die Angst vor einer Infektion mit Covid-19? Welche Auswirkungen auf die Patienten haben Sie mitbekommen? Welche Maßnahmen für zukünftige ähnliche Situationen wären aus Ihrer Sicht wichtig? Gab es „besonders eindrucksvollen Situationen“?) und aus Aussagen, die auf einer 11-stufigen Ratingskala zwischen -5 „verringert“ über 0 "gleich geblieben“ bis 5 ,vermehrt“ bewertet werden sollten (Während der „Corona-Zeit" hat sich: meine Arbeits- belastung insgesamt, die Intensität der Gespräche, die Anzahl der Neuanmeldungen, die Anzahl der Folgekontakte pro Ratsuchenden, die Planbarkeit meiner Arbeit, meine Arbeitszufriedenheit insgesamt ...).

\section{Ergebnisse}

Insgesamt nahmen 49 Frauen und 5 Männer an der Studie teil. Jeweils 27 waren in der Altersgruppe unter und über 50 Jahren. Die Berufserfahrung als Psychoonkologinnen war bei 29 kürzer und bei 25 länger als 10 Jahre. Es arbeiteten 29 in einem Akutkrankenhaus, 20 in einer Krebsberatungsstelle (KBS) und 5 in einer sonstigen Einrichtung. Der Studienabschluss war 34-mal Psychologie, 10mal Sozialarbeit/-pädagogik und 10-mal ein sonstiger Abschluss.

Im ersten Schritt der Auswertung wurden die Angaben der Mitarbeiterinnen aus Akutkrankenhäusern mit denen von KBS verglichen. Während im Akutkrankenhaus $17 \%$ davon berichteten, teilweise im Homeoffice gearbeitet zu haben, sind dies in den KBS mit $55 \%$ signifikant

ATO: Arbeitsgemeinschaft der Tumorzentren, Onkologischen Schwerpunkte und Arbeitskreise in Baden-Württemberg 


\section{Sektion B}

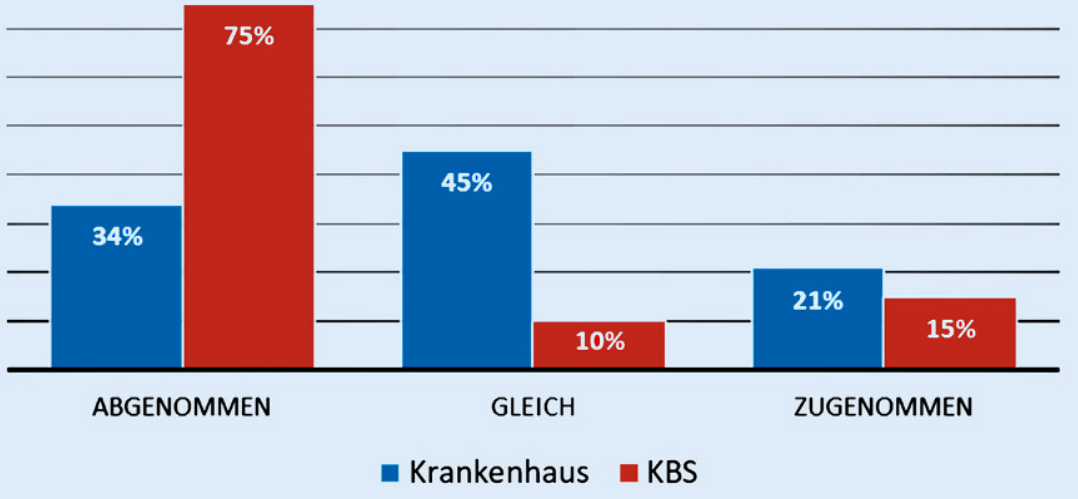

Abb. 1 \ Häufigkeitsverteilung der Angaben von 54 Psychoonkologinnen zur Veränderung der Planbarkeit ihrer Arbeit. KBS Krebsberatungsstelle

mehr. Im Krankenhaus berichteten $45 \%$ der Befragten, dass ihre Arbeitsbelastung während der Corona-Zeit abgenommen habe und $41 \%$ von einer Zunahme. In den KBS berichten $10 \%$ von einer Verringerung und $85 \%$ von einer Zunahme der Arbeitsbelastung. Auch die Planbarkeit der Arbeit unterscheidet sich, wie in - Abb. 1 zu sehen, signifikant.

Die Intensität der Gespräche (59\% höher, 6\% niedriger), die Anzahl der Neuanmeldungen ( $9 \%$ mehr, $69 \%$ weniger) und Folgekontakte $(56 \%$ mehr, $7 \%$ weniger) sowie die Arbeitszufriedenheit (20\% höher, $53 \%$ verringert) zeigen dahingegen keine signifikanten Unterschiede zwischen Akutkrankenhaus und KBS. Auch bei einer Kategorisierung der offenen Frage „Wie ist es ihnen ganz persönlich bei der Arbeit ergangen" (persönliches Erleben) finden sich keine signifikanten Unterschiede (32\% eher positiv, $18 \%$ neutral und $50 \%$ eher negativ).

In den folgenden Berechnungen wurden die Zusammenhänge zwischen dem persönlichen Erleben und den anderen erfassten Merkmalen der Teilnehmer untersucht. Es fanden sich signifikante Korrelationen mit geringer Planbarkeit $(p=0,03)$ und geringer Arbeitszufriedenheit $(p<0,01)$, dahingegen keine relevanten Zusammenhänge mit der Intensität, Dauer und Anzahl der Gespräche. Auch die Länge der Berufserfahrung und Homeoffice zeigten keine relevanten Zusammenhänge mit dem persönlichen Erleben. Psychoonkologinnen über 50 Jahre berichteten jedoch seltener, dass es ihnen bei der Arbeit negativ ergangen sei, als ihre jüngeren Kolleginnen ( $24 \%$ vs. $56 \%$ ).

In den Antworten auf die offenen Fragen wird häufig von einer großen Verunsicherung sowohl aufseiten der Patienten und Angehörigen wie auch bei den Beratungskräften berichtet. Die Umstellung der Beratungen auf telefonische Kontakte, die zu treffenden Schutzmaßnahmen und Homeoffice waren neue Erfahrungen.

Bei der Frage nach der Rolle der Angst vor einer Infektion mit Covid19 bei Krebspatienten aus Sicht der behandelnden Psychoonkologinnen finden sich deutliche Unterschiede innerhalb der Antworten. Eine Hälfte der Psychoonkologinnen berichtet von „einer großen, bisweilen zentralen Rolle“, die andere Hälfte von einer ,eher untergeordneten Rolle“. „Teilweise haben sich Patienten von Ängsten vor Covid-19 bewusst abgegrenzt, da sie mit ihrer Krebserkrankung schon genug Belastung hatten."

In der Frage nach den Auswirkungen der Beschränkungen auf die Patienten wurde aufgeführt, dass gerade frisch diagnostizierte, in Behandlung befindliche und palliative Tumorpatienten in dieser Zeit zusätzlich erheblichen Belastungen ausgesetzt gewesen seien. Es bestehe eine „doppelte Krise“ durch eingeschränkte bzw. verzögerte medizinische Versorgung und den Wegfall wichtiger Ressourcen. „Zusätzlich zur Belastung durch die Krebserkrankung kam die Einsamkeit durch Besuchsverbote in der Klinik und Kontaktbeschränkungen“.
Berichtet wurde auch von Ärger und Wut der Patienten über Einschränkungen der individuellen Freiheiten, gefährdende Verhaltensweisen und mangelhafte Erklärungen für die Maßnahmen. Verunsicherungen bestünden dadurch, dass die zuvor bestandene Sicherheit durch das Medizinsystem verloren gegangen sei. Einsamkeit, Verlassenheit und Verzweiflung bis hin zum Todeswunsch wurden genannt. Es fehlten nicht mehr mögliche Aktivitäten und ablenkende Ressourcen. Zudem bestand die Sorge über eine mögliche Ansteckung im Krankenhaus, aber auch über die Gesundheit der Angehörigen. „Das Leben der gesamten Familie verändert sich, Kinder haben Angst, Elternteil anzustecken, bleiben zu Hause, treffen sich weniger mit Freunden“. Dahingegen wurden nur wenig positive Aspekte genannt: ein durch Lockdown geringeres Infektionsrisiko sowie eine Entlastung durch den Wegfall von sozialen Anforderungen und Terminen.

Hinsichtlich der Situation der Angehörigen von Krebspatienten wurde aus Sicht der Psychoonkologinnen von Stress, Ärger und Unverständnis berichtet, da sie die Patienten bei Untersuchungen und teilweise auch im Sterbeprozess allein lassen mussten.

$\mathrm{Zu}$ eigenen Erfahrungen der Psychoonkologinnen wurde über unterschiedliche Aspekte berichtet. Neben positiven Aspekten: „Für mich waren es häufig intensivere und zufriedenstellendere Begegnungen in den Beratungen" oder „Ich war froh, einen systemrelevanten Arbeitsplatz zu haben" waren andere Aspekte eher negativ: „Ich fand es sehr anstrengend. Es waren unendlich viele Telefonate. Abends waren alle Worte aufgebraucht."

Bezüglich der Kommunikation zwischen Psychoonkologin und Patient wurden überwiegend negative Aspekte genannt: „Der Mundschutz stört, Atembeschwerden, Mimik fehlt", „Es war schwer die eigene Meinung zum Thema [Corona] nicht mit der des Patienten zu vermischen“, „Insbesondere [war die CoronaThematik] in den ersten Wochen eine störende ,Nebenspur' bei mir [die von den onkologischen Themen ablenkte]" und 
„die Sorge vor Ansteckung und Verbreitung des Virus an Hochrisikopatienten“.

Folgende Wünsche für zukünftige Strategien in ähnlichen Situationen wurden geäußert: „durch proaktive Kommunikation mehr Sicherheit für [onkologische] Patienten“, „individuellere Besuchsregelungen, z.B. Konzept für menschenwürdiges Abschiednehmen und Sterben“, „schlüssige Sicherheitskonzepte“, „ausreichende technische Möglichkeiten“, „Schutzmaterialien für wichtige persönliche Kontakte" und „Konzepte für die Unterstützung der Angehörigen“.

\section{Diskussion und Schlussfolgerungen}

Unsere Erhebung beansprucht nicht, repräsentativ oder vollständig zu sein. Jedoch stellt sie eine breite Auswahl an Erfahrungen von Psychoonkologinnen während der ersten Corona-Phase dar.

Die Variable des persönlichen Erlebens werten wir als zentrales Kriterium für eine langfristige Zufriedenheit mit der eigenen Arbeit. Diese zeigte keinen Zusammenhang mit der Intensität oder der Art der Patientengespräche, jedoch mit der Planbarkeit der Arbeit. Zusammen mit den häufig geäußerten Wünschen nach einer guten multimedialen Ausstattung und Schutzausrüstungen zeichnen sich Vorschläge für ein Vorgehen bei künftigen ähnlichen Szenarien $\mathrm{ab}$.

\section{Fazit für die Praxis}

- Mitarbeiter der Leitungsebene sollten sich mit den Mitarbeitern der Beratungsteams zusammensetzen und geplante Maßnahmen besprechen. Hierdurch werden Konzepte kommuniziert und somit für alle Beteiligten gleichermaßen verständlich.

- Eine sehr gute multimediale Ausstattung zum Führen von Videogesprächen (mobile Notebooks, Kamera, Mikrofon, Breitband-Internetverbindung) und Telefonaten (Headset) ist selbstverständlich zur Verfügung zu stellen.

- Bei bestimmten Gruppen von Patienten und Ratsuchenden (z. B. stationär, sehr hoch belastet, vereinsamt) werden weiterhin persönliche Kontakte als notwendig erachtet. Hierfür braucht es eine gute Ausstattung mit persönlicher Schutzausrüstung.

- Wir empfehlen die genannten Punkte zu berücksichtigen. Sie werden kurzfristig zu einer höheren Arbeitszufriedenheit im Team und auf den eigentlichen Inhalt fokussierten Gesprächen (ohne Ansteckungsängste und Unterbrechungen wegen schlechter technischer Ausstattung) führen. Mittelfristig wird dies zu einem stabileren Behandlungsteam (höhere Arbeitszufriedenheit) und einer effizienteren Versorgung von Patienten und Ratsuchenden führen (keine langen Vorbereitungen, keine Arbeitszeitverschwendung durch technisch bedingte Probleme). Auch langfristig werden diese Maßnahmen zu mehr Ratsuchenden in Krebsberatungsstellen (Gespräche für Ratsuchende, die kein persönliches Gespräch wünschen, immobil sind oder eine lange Anfahrt zu Einrichtung haben) und einem besseren „Ruf" der Einrichtung (positive Erfahrungen, flexibel, modern) führen.

\section{Korrespondenzadresse}

Dr. Dipl.-Psych. Andreas Ihrig

Psychosoziale Krebsberatungsstelle

Nordbaden, Sektion Psychoonkologie, Klinik für Allgemeine Innere Medizin und Psychosomatik, Universitätsklinikum Heidelberg INF 155, Heidelberg, Deutschland andreas.ihrig@med.uni-heidelberg.de

\section{Neue Krebsdiagnostik: Blick in den Tumor in 3D}

Bisher analysierte man Tumore nur anhand dünner Schnitte. An der TU Wien in Zusammenarbeit mit der TU München wurde nun eine Technik entwickelt, die erstmals ganze Stücke des Tumors in 3D sichtbar macht.

Nach einer Krebsoperation ist die entscheidende Frage: Sind möglicherweise Krebszellen zurückgeblieben, die weiterwachsen können, oder wurde tatsächlich der gesamte Tumor entfernt? Um das herauszufinden, wird der Tumor in der Pathologie untersucht. Bisher fertigte man dünne Schnitte an, die dann unter dem Mikroskop analysiert wurden. Eine neue Technik, entwickelt an der TU Wien, gemeinsam mit der TU München, soll nun eine Revolution in der Pathologie einleiten: Es gelang, Tumorgewebe durchsichtig zu machen und mit einem speziellen Ultramikroskop zu durchleuchten. So kann man ganz ohne Schnitt das gesamte entnommene Gewebe in 3D analysieren. Die Zuverlässigkeit der Diagnose soll dadurch deutlich gesteigert werden.

\section{Durchsichtiges Gewebe:} Ultramikroskopie

Mit Hilfe einer speziellen Technik, der sogenannten Ultramikroskopie, ist es nun aber möglich, den ganzen Tumor dreidimensional sichtbar zu machen daran arbeitete Inna Sabdyusheva im Rahmen ihrer Dissertation (universitätsübergreifend, an der TU Wien und dem Zentrum für Hirnforschung der MedUni Wien). Sie entwickelte ein chemisches Verfahren, mit dem man BrustkrebsProben „klären“ kann - sie werden durchsichtig, die Struktur bleibt aber unverändert, und die Krebszellen sind nach wie vor zu erkennen.

Originalpublikation: I. Sabdyusheva Litschauer et al (2020) 3D histopathology of human tumours by fast clearing and ultramicroscopy, Scientific Reports 10, 17619. www.nature.com/articles/s41598 020-71737-w

Quelle: Technische Universität Wien 
Hier steht eine Anzeige.

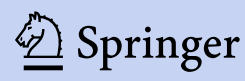

